World J Surg. 2012 September ; 36(9): 2074-2079. doi:10.1007/s00268-012-1629-6.

\title{
Ratio of Cesarean Sections to Total Procedures as a Marker of District Hospital Trauma Capacity
}

\author{
Robin T. Petroze, \\ Department of Surgery, University of Virginia Health System, P.O. Box 800679, Charlottesville, \\ VA 22908, USA; Department of Surgery, Faculty of Medicine, National University of Rwanda, \\ Butare, Rwanda \\ Winta Mehtsun, \\ University of Virginia, Charlottesville, VA, USA \\ Albert Nzayisenga, \\ Department of Orthopedic Surgery, King Faisal Hospital, Kigali, Rwanda \\ Georges Ntakiyiruta, \\ Department of Surgery, Faculty of Medicine, National University of Rwanda, Butare, Rwanda \\ Robert G. Sawyer, and \\ Department of Surgery, University of Virginia Health System, P.O. Box 800679, Charlottesville, \\ VA 22908, USA \\ J. Forrest Calland \\ Department of Surgery, University of Virginia Health System, P.O. Box 800679, Charlottesville, \\ VA 22908, USA
}

\begin{abstract}
Background-There are few established metrics to define surgical capacity in resource-limited settings. Previous work hypothesizes that the relative frequency of cesarean sections (CS) at a hospital, expressed as a proportion of total operative procedures (\%CS), may serve as a proxy measure of surgical capacity. We attempted to evaluate this hypothesis as it specifically relates to hospital capacity for emergency interventions for injury.
\end{abstract}

Methods-We conducted a WHO survey of emergency surgical capacity at 40 Rwandan district hospitals in November 2010 and extracted annual operative volume for 2010 from the Ministry of Health centralized statistical system. We dichotomized the 40 hospitals into low and high \%CS groups below and above the median proportion of CS performed. We compared low and high $\% \mathrm{CS}$ groups across self-reported capabilities related to facility characteristics, trauma supplies, procedural capacity, and surgical training using bivariate $\chi^{2}$ statistics with significance indicated at $p \leq 0.05$. We evaluated herniorrhaphy proportion of total procedures (\%Hernia) as a representative general surgery procedure in the same manner.

Results-High \%CS hospitals were less likely to report capability related to blood banking ( $p=$ $0.05)$, amputation $(p=0.04)$, closed fracture repair $(p=0.04)$, inhalational anesthesia $(p=0.05)$, and chest tube insertion $(p=0.05)$. Availability of reliable electricity was the only measure that showed statistical significance with the \%Hernia measure $(p=0.02)$.

C Société Internationale de Chirurgie 2012

R. T. Petrozertp3z@virginia.edu.

Previously presented at: Annual General Meeting, College of Surgeons of East, Central, and Southern Africa (COSECSA) Lusaka, Zambia, December 2011. 
Conclusions-Cesarean section proportion shows some utility as a marker for district hospital injury-care capacity in resource-limited settings.

\section{Introduction}

As the international public health community and individual healthcare systems recognize the immense need to improve surgical care in resource-limited settings, it is important to develop metrics to measure improvement in surgical capacity [1-4]. Limited reliable data exist on the burden of surgical disease; less than $70 \%$ of World Health Organization (WHO) member states have data on surgical procedures performed, and even fewer on surgical outcomes [5].

The WHO Global Initiative for Emergency and Essential Surgical Care (GIEESC) situational analysis tool for emergency and essential surgical capacity provides a framework for quantifying hospital-level infrastructure, human resources, procedural interventions, and emergency and resuscitative supplies [6,7]. Whereas the WHO tool has been used to evaluate surgical capacity in more than 30 countries, the tool is predominantly qualitative and subjective. This can provide the background information needed to plan interventions for increasing surgical capacity but makes it difficult to use as an indicator of capacity [8]. Scarcity of materials and a shortage of surgical personnel in resource-limited settings make surgical outcomes assessments difficult. In effect, there are no duplicable, objective measures of surgical capacity being used in resource-limited settings to track improvements.

A simple indicator to assess surgical capacity in resource-limited settings has been proposed by Drs. Kushner, Groen, and Kingham [9]. Using available nationwide procedure data in several sub-Sahara African nations, they found that the percentage of cesarean sections (CS) to total operations (\%CS) was much higher for low-income countries than for high-income countries. They hypothesized that as surgical capacity improves, a wider range of procedures are performed and the \%CS goes down. In high-income countries, for example, CS comprise $2.6 \%$ of total procedures. They proposed the utilization of \%CS as a proxy indicator for overall adequacy of emergency and essential surgical care [9].

The proposed \%CS method has yet to be tested. This study in Rwanda aims to utilize capacity measures in the WHO tool to validate the use of the \%CS surgical capacity indicator. Our hypothesis is that the relative proportion of CS performed at the district hospital level is inversely proportional to district hospital-level capacity for emergency interventions for trauma and therefore a useful measure for tracking improvements in emergency surgical capacity.

\section{Methods}

We conducted a nationwide survey utilizing the WHO tool in November 2010 at 40 Rwandan district hospitals, where the primary surgical provider is a general practitioner. We extracted annual operative volume for 2010 from the Ministry of Health (MOH) centralized statistical system (Health Management Information Systems-HMIS). Procedures are reported monthly in defined categories, with each hospital being subject to audits by the $\mathrm{MOH}$ to ensure reporting accuracy. From the total operative volume, we could calculate the cesarean section proportion of total procedures (\% CS). We also evaluated herniorrhaphy proportion of total procedures as a representative general surgery procedure (\%Hernia). $\%$ Hernia is an inclusive hernia category as defined by the Rwanda HMIS system; it does not differentiate inguinal versus abdominal/umbilical hernia repair. 
Rwanda does not have a parallel private hospital system, and so this study includes all district hospitals in the country. Referral hospitals $(n=3)$ were excluded. Each hospital level in the Rwandan healthcare system has a minimum package of services offered; CS and herniorrhaphy are included in the minimum package for district hospitals.

We dichotomized the 40 hospitals into low and high \%CS groups above and below the median proportion of CS performed. The same was done for low and high hernia groups (\%Hernia). We compared low and high \%CS groups and low and high \%Hernia groups across self-reported capabilities related to facility characteristics, trauma supplies, procedural capacity, and surgical training using bivariate chi-squared statistics. A $p$ value $\$ 0.05$ was used to indicate significance.

\section{Results}

Full results from the comprehensive survey utilizing the WHO tool have been previously reported [10]. Amongst the high \%CS group, the average proportion of CS performed compared with other types of procedures was $77.9 \%$, compared with $49.7 \%$ in the low $\% \mathrm{CS}$ group. Among the high \%Hernia group, the average proportion of hernias performed was $7.2 \%$ compared with $2.4 \%$ in the low \%Hernia group. Figure 1 details the range of $\% \mathrm{CS}$ and \%Hernia performed.

Table 1 shows results for facility capacity. High \%CS hospitals were less likely to report capability related to blood banking $(p=0.05)$. High \%Hernia hospitals were more likely to report reliable electricity sources $(p=0.02)$. Table 2 evaluates self-reported facility emergency interventions and shows several significant differences in the \%CS groups but none in the \%Hernia group. Hospitals with a low \% CS were more likely to perform amputation $(p=0.04)$, inhalational anesthesia $(p=0.05)$, closed fracture repair $(p=0.04)$, and chest tube insertion $(p=0.05)$, with a trend toward significance with laparotomy $(p=$ $0.07)$ and emergency airway $(p=0.06)$. No statistically significant differences were found for either \%CS or \%Hernia when we evaluated representative facility supplies (Table 3).

\section{Discussion}

Our study sought to evaluate a proposed metric [9] for evaluating surgical capacity in lowresource settings. There is a need for simple, reliable, and duplicable metrics for measuring surgical development in areas where surgical resources and providers are scarce [11, 12]. The \%CS metric is not meant to be a substitute for other metrics such as prospective registries, detailed and centrally reported operative logs, and surgical outcomes tracking, such as postoperative mortality, functional outcomes, and surgical infections. However, these measures can be expensive and time-intensive; in many resource-limited settings, detailed surgical data are extremely limited, outcomes are not tracked, and funding for surgical development is negligible [13]. At the district hospital level, where the primary surgical and obstetric provider usually is a generalist and specialty care is not available, a CS is an emergency procedure and often the most common surgical procedure performed. Efforts to quantify and improve maternal mortality are a key element of the Millennium Development Goals $[14,15]$, and so CS are not only widely performed but also widely recorded.

Particularly as regards district hospital interventions for trauma (Table 2), we have shown that utilizing the \% CS can provide useful information. Hospitals with a low \%CS were more likely to perform amputation, inhalational anesthesia, closed fracture repair, and chest tube insertion, with a trend toward significance with laparotomy and emergency airway. Of interest, all of these procedures are generally considered to be core capabilities in the care of 
acute injury, a leading worldwide cause of preventable death. Open fracture repair rarely was performed at the district hospitals, and wound debridement was performed at almost all hospitals, so these interventions did not show significant differences. The ability to detect significance in our study was further limited by the small sample size of 40 district hospitals; a more robust analysis, including multivariate analysis, might be possible with a larger sample size or a sample that included multiple healthcare systems. It is important to note that a hospital's ability to perform a certain procedure in this study was self-reported by doctors and nurses in the operating room.

Several challenges exist in proposing the \%CS as a metric of surgical capacity. This metric is very dependent on birth rate, CS percentage of live births, and local and national policies, which may complicate its use in comparing regions or countries or in defining what \%CS signifies adequate capacity. In Rwanda, the national CS rate for live births was reported to be $3 \%$ in 2005, with the urban rate in Kigali being four times the rural CS rate [16]. Perhaps due to increased training and health facility access for deliveries, the national CS rate for live births had increased to $7.1 \%$ by 2010 [17]. We were not able to evaluate changes in the \%CS metric as the Rwandan HMIS system only has surgical volumes in HMIS since 2008. However, this metric most likely has its greatest utility in evaluating progress over time in a particular facility and deserves further analysis in this regard.

Our survey evaluates district hospitals where the primary surgical provider is a general practitioner; the majority of the specialists in Rwanda (surgeons, obstetricians) practice in the referral hospitals. As surgical capacity increases and district hospitals acquire trained surgeons and obstetricians, the use of \% CS may not adequately report overall surgical capacity. For that reason, we chose to evaluate a general surgery procedure that also is part of the minimum package of services mandated for the district hospitals in Rwanda (and thus, reported within HMIS) compared with \%CS. The only significant association identified with $\%$ Hernia was that hospitals with a regular electricity supply were more likely to perform more herniorrhaphies. This may relate to the increased capacity and elective nature of many hernia repairs. The ability to perform elective procedures may be limited by number and training of personnel, hospital capacity for nonemergencies, health-seeking behaviors, and materials. Ultimately, hernias represented a small percentage of total procedures (7.2 and 2.4 $\%$ for high and low \%Hernia, respectively), which may have limited our ability to draw inferences. Overall, \%CS was deemed to be more effective as an indicator of emergency surgical capacity at the district hospital level in Rwanda than \%Hernia.

\section{Conclusions}

Cesarean section proportion of total procedures (\%CS) shows some utility as a marker for district hospital injury-care capacity in resource-limited settings. As surgical capacity in countries, such as Rwanda improves, the ability to measure improvement has significant implications in funding, planning, and education. A simple tool like \%CS can be used to quantify change within a healthcare facility, but better metrics need to be developed and implemented for quantifying and comparing surgical capacity in resource-limited settings around the world.

\section{Acknowledgments}

The authors thank the Ministry of Health (MOH) of Rwanda, particularly Dr. Vincent Rusanganwa and the HMIS division, for assistance in implementation of the survey and access to surgical volumes at the district hospitals. Work was conducted in Rwanda with ethical approval from the University Teaching Hospital-Kigali. Work was funded by a Fogarty International Clinical Research Fellowship, National Institutes for Health and International Clinical Research Fellows Program at Vanderbilt University, USA (R24 TW007988). 


\section{References}

1. Farmer PE, Kim JY. Surgery and global health: a view from beyond the OR. World J Surg. 2008; 32(4):533-536. [PubMed: 18311574]

2. Ozgediz D, Riviello R. The "other" neglected diseases in global public health: surgical conditions in sub-Saharan Africa. PLoS Med. 2008; 5(6):e121. [PubMed: 18532875]

3. Bickler SW, Spiegel DA. Global surgery—defining a research agenda. Lancet. 2008; 372(9633):90_ 92. [PubMed: 18582930]

4. Weiser TG, Makary MA, Haynes AB, et al. Standardised metrics for global surgical surveillance. Lancet. 2009; 374(9695):1113-1117. [PubMed: 19782877]

5. Weiser TG, Regenbogen SE, Thompson KD, et al. An estimation of the global volume of surgery: a modelling strategy based on available data. Lancet. 2008; 372(9633):139-144. [PubMed: 18582931]

6. World Health Organization (WHO). [Accessed 24 Nov 2009] situational analysis tool. www.who.int/entity/surgery/publiations/QuickSitAnalysisEESCsurvey.pdf

7. Kingham TP, Kamara TB, Cherian MN, et al. Quantifying surgical capacity in Sierra Leone: a guide for improving surgical care. Arch Surg. 2009; 144(2):122-127. (discussion 128). [PubMed: 19221322]

8. Osen H, Chang D, Choo S, et al. Validation of the world health organization tool for situational analysis to assess emergency and essential surgical care at district hospitals in Ghana. World J Surg. 2011; 35(3):500-504. [PubMed: 21190114]

9. Kushner AL, Groen RS, Kingham TP. Percentage of cesarean sections among total surgical procedures in sub-Saharan Africa: possible indicator of the overall adequacy of surgical care. World J Surg. 2010; 34(9):2007-2008. [PubMed: 20517607]

10. Petroze RT, Nzayisenga A, Rusanganwa V, et al. Comprehensive national analysis of emergency and essential surgical capacity in Rwanda. Br J Surg. 2012; 99(3):436-443. [PubMed: 22237597]

11. Ozgediz D, Kijjambu S, Galukande M, et al. Africa's neglected surgical workforce crisis. Lancet. 2008; 371(9613):627-628. [PubMed: 18295007]

12. Ozgediz D, Hsia R, Weiser T, et al. Population health metrics for surgery: effective coverage of surgical services in low-income and middle-income countries. World J Surg. 2009; 33(1):1-5. [PubMed: 18958518]

13. Kruk ME, Wladis A, Mbembati N, et al. Human resource and funding constraints for essential surgery in district hospitals in Africa: a retrospective cross-sectional survey. PLoS Med. 2010; 7(3):e1000242. [PubMed: 20231869]

14. Rasch V. Maternal death and the Millennium Development Goals. Dan Med Bull. 2007; 54(2): 167-169. [PubMed: 17521538]

15. Pitt C, Greco G, Powell-Jackson T, Mills A. Countdown to 2015: assessment of official development assistance to maternal, newborn, and child health, 2003-08. Lancet. 2010; 376(9751):1485-1496. [PubMed: 20850869]

16. Ministry of Health (MOH) [Rwanda] NISR. ICF Macro. Rwanda demographic and health survey 2005. MOH, NISR, and ICF Macro; Calverton: 2006.

17. National Institute of Statistics of Rwanda (NISR) [Rwanda]. Ministry of Health (MOH) [Rwanda]. ICF International. Rwanda Demographic and Health Survey 2010. NISR, MOH, and ICF International; Calverton: 2011. 

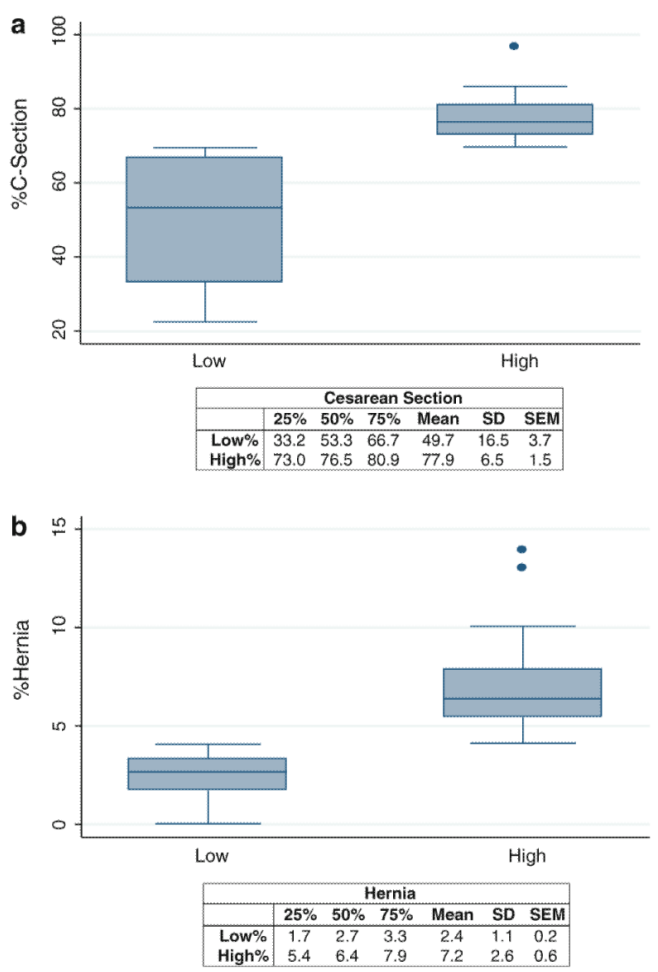

Fig. 1.

a Cesarean section range, Rwandan District Hospitals $(n=40)$, b herniorrhaphy range, Rwandan district hospitals $(n=40)$ 


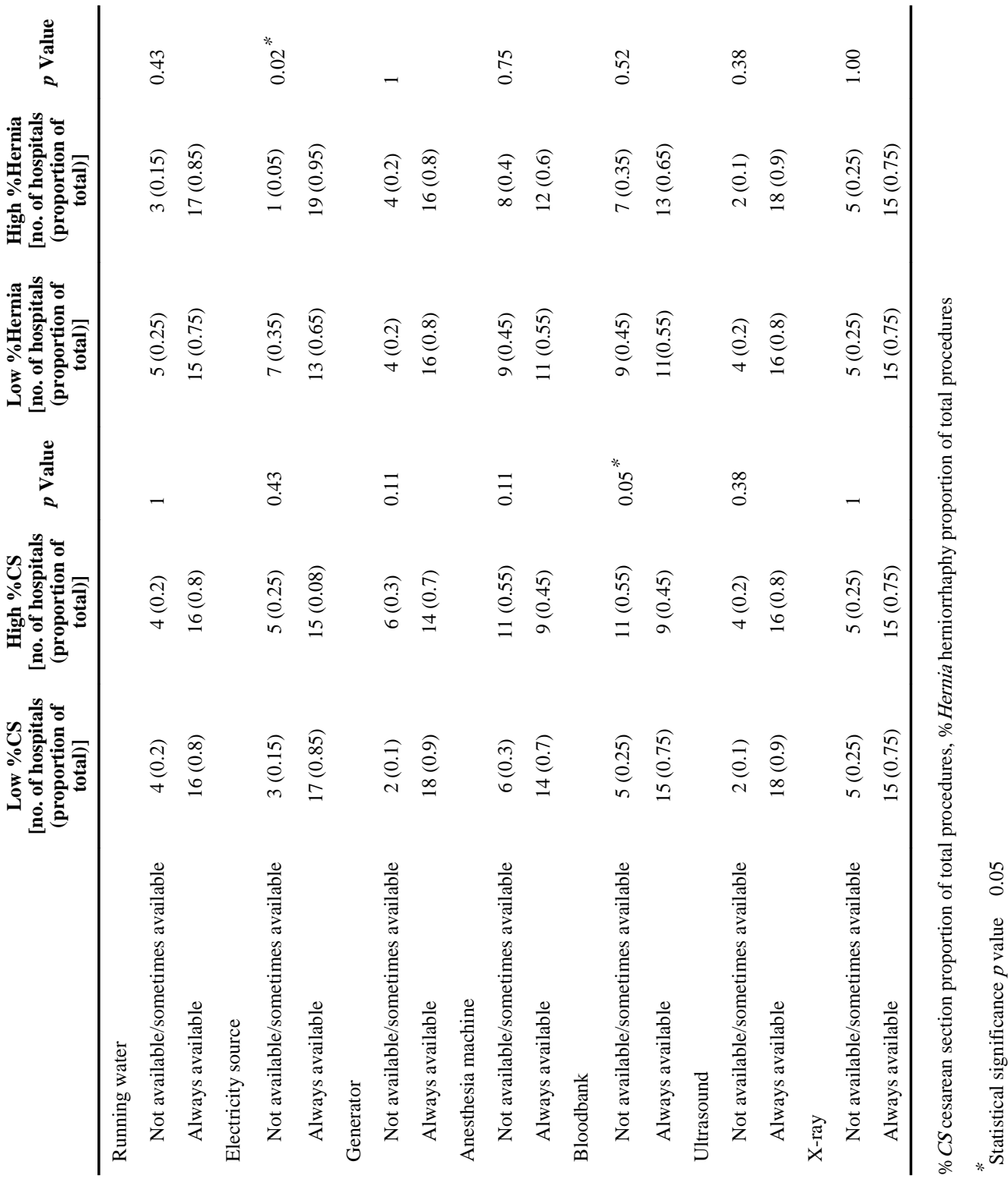




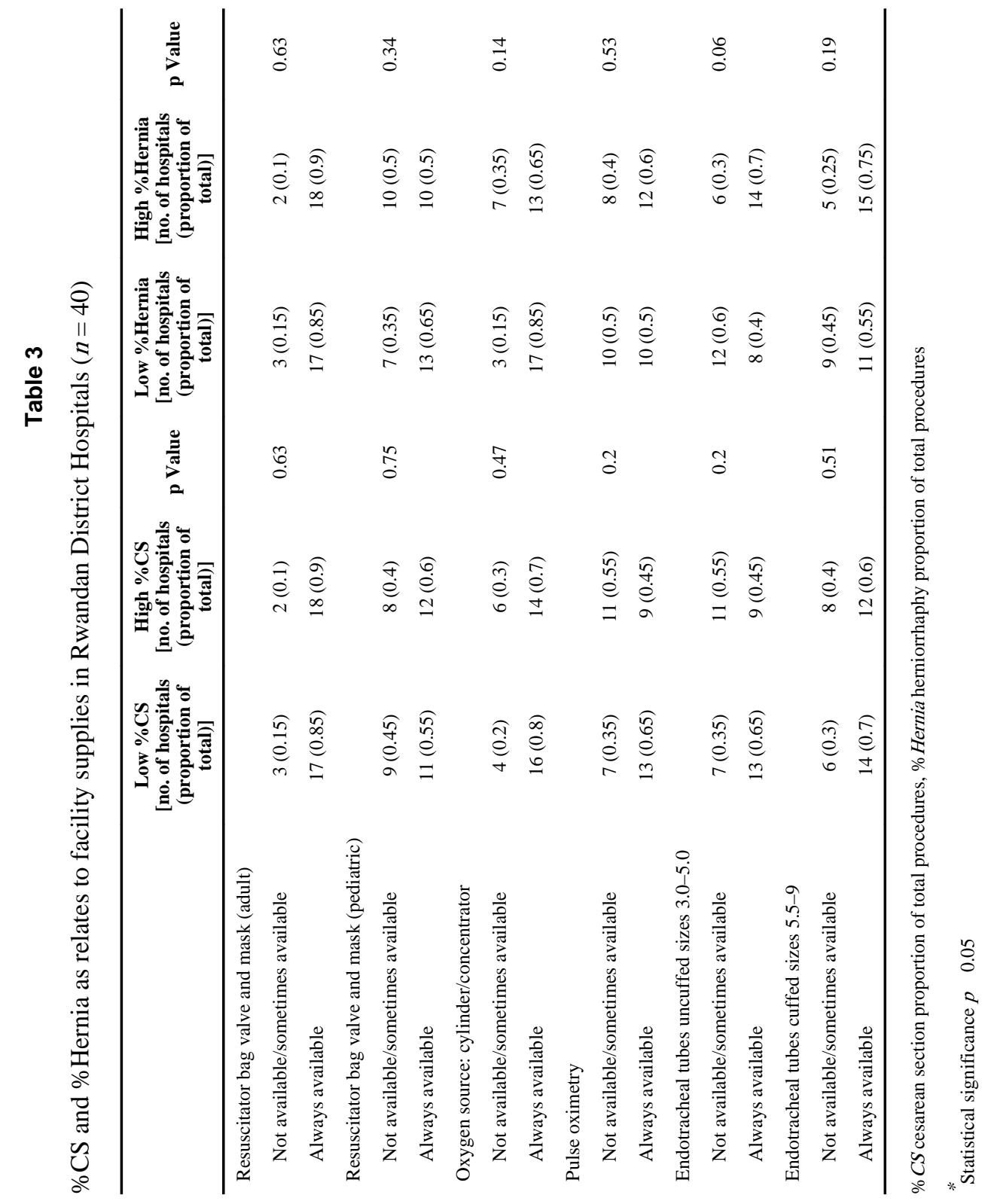

World J Surg. Author manuscript; available in PMC 2013 September 01. 